\title{
HPV E6 antisense induces apoptosis in CaSki cells via suppression of E6 splicing
}

\author{
Cheong Weon $\mathrm{Cho}^{1 *}$, Haryoung Poo ${ }^{1 *}$, \\ Young Sik Cho', Min Chul Cho', \\ Kyung Ae Lee ${ }^{1}$, Shin Je Lee ${ }^{1,2}$, \\ Sue Nie Park ${ }^{1,3}$, In Ki Kim ${ }^{4}$, \\ Yong Keun Jung ${ }^{4}$, Yong Kyung Choe', \\ Young II Yeom ${ }^{1}$, In Seong Choe ${ }^{1}$ \\ and Do Young Yoon ${ }^{1,5}$
}

${ }^{1}$ Division of Life Science, Korea Research Institute of Bioscience and Biotechnology, P. O. Box 115, Yuseong, Taejon 305-600;

${ }^{2}$ Department of Microbiology, College of Medicine, Soonchunhyang Univ, ChunAn, Korea,

${ }^{3}$ Division of Viral Products, Korea Food and Drug Administration, \#5 Nokbun dong, Eunpyung-gu, Seoul 122-704, Korea

${ }^{4}$ Department of Life Science, Kwangju Institute of Science and Technology, Kwangju 500-712, Korea

${ }^{5}$ Corresponding author: Tel, +82-42-860-4218;

Fax, +82-42-860-4593; E-mail; dyyoon @ mail.kribb.re.kr

* These two authors contributed equally to this work.

Accepted 14 may 2002

Abbreviations: HPV, human papillomavirus; E6(AS), E6 antisense nucleic acid; E6(S), E6 sense nucleic acid; MPT, mitochondrial permeability transition; PCR, polymerized chain reaction

\begin{abstract}
Cervical cancer is known to be highly associated with viral oncogene E6 and E7 of human papilloma virus. Down-regulation of oncogene expression by antisense-based gene therapy has been extensively studied. To investigate the effect of HPV 16 E6 antisense nucleic acid (AS) on cervical cancer cells, human cervical cancer cell lines, CaSki and SiHa cells harboring HPV 16 genome were transfected with plasmid containing E6(AS). The decreased viability and the apoptotic morphology were observed in E6(AS)- transfected cervical cancer cell lines. By 6 h after transfection, inhibition of E6 splicing, rapid upregulations of p53 and a p53-responsive protein, GADD45, were displayed in E6(AS)-transfected CaSki cells. Furthermore, E6(AS) induced loss of mitochondrial transmembrane potential, release of mitochondrial cytochrome $c$ into the cytoplasm, and subsequent activation of caspase-9 and caspase-3. These results indicate that HPV 16 E6(AS) induces
\end{abstract}

apoptosis in CaSki cells via upregulation of p53 and release of cytochrome c into cytoplasm, consequently activating procaspase-9 and procaspase-3.

Keywords: apoptosis; antisense; HPV E6; p53; E6 splicing: mitochodrial potential transition; cytochrome c; caspase

\section{Introduction}

Cervical cancer has been reported to be highly associated with viral oncogene E6 and E7 of human papillomavirus (HPV). There have been abundant experimental evidences indicating that the E6 and E7 oncogenes of HPV play major roles in the development as well as in the maintenance of the malignant phenotype of cervical carcinoma. E6 and E7 of HPV 16, the most frequently associated type of HPV with cervical carcinomas, play a role to induce malignant tumor by degradation of the cellular anti-tumor proteins, p53 and retinoblastoma $(\mathrm{Rb})$, respectively in cervical cells (Sheffner et al., 1991). E6 protein forms complexes with p53 (Storey et al., 1998) and abrogate p53 function of transcriptional activation by stimulating its degradation via ubiquitin-mediated proteolysis in an E6-associated protein (E6AP) mediated reaction, resulting in tumor progression (Lechner et al., 1994; Beer-Romero et al., 1997).

Hence, E6 oncogene is one of the ideal targets for directed anti-gene therapy in cervical cancer (Madrigal et al., 1997). The repressed level of oncogene expression through the use of anti-sense RNA or antisense oligodeoxynucleotides may offer a new way to modify some genetic traits at the somatic level controlled by these oncogenes and possibly related to the maintenance of the transformed phenotype (Paoletti, 1988). One of these approaches is to develop an antisense and/or ribozyme strategy to inhibit E6 and E7 gene expression in cervical cancers to reverse the malignant phenotype (He and Huang, 1997). Antisense oligonucleotides of $\mathrm{E} 6$ inhibited tumor cell growth in vitro and in vivo by down-regulation of E6 gene expression (Tamura et al., 1995). The proliferation of HPV 16 or 18 positive cervical cancer cells is inhibited by oligonucleotides expressing RNA antisense targeting to E6 which is associated with carcinogenesis and apoptosis (programmed cell death). However, the mechanism of growth inhibition induced by E6 antisense has not been clearly understood (Hamada et al., 1996). The 
mitochondrial permeability transition (MPT) is required for the activation of apoptotic cell death and a loss in the mitochondrial potential $(\mathrm{m})$ is induced by the change of permeability associated with MPT (Heiskanen et al., 1999). The opening of MPT pores, located on the inner mitochondrial membrane, causes the loss of mitochondrial membrane potential which leads subsequently to the release of cytochrome $\mathrm{c}$ from mitochondria to cytoplasm. Caspase- 9 and caspase- 3 are sequentially activated, which in turn activate downstream death substrates and endonucleases resulting in the apoptotic nuclear morphology (Heiskanen et al., 1999; Sun et al., 1999). In current study, the apoptotic effect induced by HPV 16 E6 antisense nucleic acid [E6(AS)] and its mechanism were examined in cervical cancer cells. Mammalian expression vectors expressing the antisense genes directed against HPV 16 E6 gene were constructed and the antisense constructs were delivered into cervical cancer cell lines, $\mathrm{SiHa}$ and CaSki cells. The influence of HPV E6 antisense on E6 splicing pattern in CaSki cells and on cell growth, the level of p53, mitochondrial permeability transition, mitochondrial cytochrome c release into cytoplasm and activation of caspase- 3 and caspase- 9 were investigated.

\section{Materials and Methods}

\section{Construction of sense and antisense nucleic acids}

E6 cDNA was obtained by PCR amplification from total RNA isolated from CaSki cell lines using following primer sets; 5'-GCG GCC GCC ACC ATG TTT CAG GAC CAC AG-3' (sense) and 5'-CTG CGG CCG CGA TTA CAG CTG GGT TTT CTC T-3' (antisense). The PCR products were inserted into cloning vector $\mathrm{PCR}^{\circledR}$ 2.1-TOPO (Invitrogen, Carlsbad, CA, USA) as an intermediate for subcloning into corresponding expression vector as described (Cho et al., 2000). In brief, E6 inserts containing Not I site was subcloned into pTarget (Promega, Madison, WI, USA), which had been prepared by digestion with Not I, in reverse and forward orientations, to construct vectors expressing E6(AS) and E6(sense), respectively.

\section{Cell culture and transfection}

Two established human cervical carcinoma cell lines, CaSki and SiHa cells containing HPV 16, were used in this study. Also two other human cervical cancer cell lines, C-33A cells which have no HPV genome and HeLa cells containing HPV 18, were used as control cells. All human cervical cancer cell lines were grown in DMEM (Dulbecco's modified Eagle's medium) supplemented with $10 \%$ FBS (Gibco BRL, Grand Island, NY, USA) in a 5\% $\mathrm{CO}_{2}$ incubator. The plasmid pTarget (the plasmid used as a cloning vector for control transfections), antisense and sense nucleic acids of E6 [E6(AS) plasmid containing DNA sequences encoding HPV 16 E6 in the antisense orientation and $E 6(S)$ containing DNA sequences encoding HPV 16 E6 in the sense orientation] were transfected into cells by Lipofectamine 2000 reagent (Life Technologies, Inc., Gaithersburg, MD, USA) according to the manufacturer's protocol. Briefly, CaSki cells were seeded into 6-well plates at a density of 5 $\times 10^{5} / \mathrm{ml}$ a day prior to experiments. After overnight, CaSki cells were washed 2 times with PBS, the DNAlipofectamine 2000 reagent complexes were prepared and incubated in $2 \mathrm{ml}$ of DMEM medium containing DNA-lipofectamine 2000 reagent complexes [250 $\mu \mathrm{l}$ of OPTI-MEM I (modified minimal Eagle's medium), $5 \mu \mathrm{l}$ of lipofectamine 2000 reagent, $5 \mu \mathrm{g}$ of DNA].

\section{Measurement of cytotoxicity}

WST-1 (Boehringer Mannheim, Mannheim, Germany) was used to measure the viability of transfected cervical cancer cells including CaSki, C-33A and HeLa cells. Cells were plated at a density of $2 \times 10^{4} / 100 \mu \mathrm{l} /$ well on a 96-well plate. Each of plasmids [E6(S), E6(AS), pTarget plasmid] were transfected using lipofectamine 2000 reagent according to the manufacturers protocol. Two days after transfection, $10 \mu \mathrm{l}$ of cell proliferation assay reagent WST-1 was added to the cells and incubated at $37^{\circ} \mathrm{C}$ for $2 \mathrm{~h}$, and then the optical density was measured at $450 \mathrm{~nm}-650 \mathrm{~nm}$.

\section{Apoptotic cell morphology}

CaSki cells were seeded in 6-well plates at a density of various number $\left(10^{5} \sim 10^{6}\right)$ of cells per well. E6(AS) or vector control (pTarget) was transfected using lipofectamine 2000 reagent into CaSki cells to observe the effect on the cell morphology. Two days after transfection, the cells were washed twice with PBS, fixed in $4 \%$ formaldehyde and stained with tryphan blue to observe the cell morphology under the light microscopy. For apoptosis analysis, the CaSki cells were transfected with E6(AS) or vector control (pTarget) and 2 days after transfection, the cells were stained with $2.5 \mu \mathrm{g} / \mathrm{ml}$ of bisbenzimide Hoechst 33258 (Sigma, USA) for $30 \mathrm{~min}$. Stained cells were examined under the fluorescence microsope (Axiovert, ZEISS, Germany).

\section{RT-PCR analysis}

The effects of E6(AS) on mRNA levels of E6 in CaSki cells were investigated. Total RNAs were extracted using the RNeasy Mini Kit (Qiagen, Hilden, Germany). cDNA was synthesized from a serial dilution of each sample in $50 \mu \mathrm{l}$ reaction volume containing $5 \mu \mathrm{l} 10 \mathrm{X}$ firststrand buffer, $1 \mu \mathrm{l}$ RNase block ribonuclease inhibitor (40 $\mathrm{U} / \mu \mathrm{l}), 2 \mu \mathrm{l} 100 \mathrm{mM}$ dNTPs and $1 \mu \mathrm{l} \mathrm{MMLV-RT} \mathrm{(50} \mathrm{U/ \mu l).}$ The mixture was incubated at $37^{\circ} \mathrm{C}$ for $1 \mathrm{~h}$ to synthesize the first strand cDNA. Then cDNAs were amplified by 
PCR following denaturation of the template (30 cycles: $1 \mathrm{~min}$ at $95^{\circ} \mathrm{C}, 1 \mathrm{~min}$ at $57^{\circ} \mathrm{C}$ and $1 \mathrm{~min} 30 \mathrm{~s}$ at $72^{\circ} \mathrm{C}$ ). The following primers were used for PCR amplification: 5'GCG GCC GCC ACC ATG TTT CAG GAC CAC AG-3' (sense) and 5'-CTG CGG CCG CGA TTA CAG CTG GGT TTT CTC T-3' (antisense) to identify the transcripts of E6. The E7 primers are 5'-GCG GCC GCC ACC ATG GCA TGG CAT GGA GAT ACA CCT3' (sense) and 5'-TTA TGG TTT CTG AGA ACA-3' (antisense). The primer sequences for $\beta$-actin as an internal standard were 5'-GTG GGG CGC CCC AGG CAC CA-3' (sense) and 5'-CTC CTT AAT GTC ACG CAC GAT TTC-3' (antisense). For a negative control, we used total cellular RNAs which were extracted both from the non-transfected cells and from mocktransfected cells. The reaction products were analyzed on $1 \%$ agarose gel. DNA bands were detected by ethidium bromide staining.

\section{Western blot}

Total cellular proteins were extracted by disrupting cells in a lysis buffer (Promega, Madison, WI, USA). Fifty micrograms of protein were boiled in sample buffer (125 $\mathrm{mM}$ Tris- $\mathrm{HCl}, \mathrm{pH} 6.8,1 \%$ SDS, $2 \% \beta$-mercaptoethanol and $0.01 \%$ bromophenol blue) for 5 minutes and then loaded onto a $12 \%$ polyacrylamide-SDS gel. After $3 \mathrm{~h}$ electrophoresis at $50 \mathrm{~V}$, the proteins were transferred from the gel to an Immobilon-P membrane (Millipore Corp., Bedford, MA, USA). The membrane was then blocked with PBS containing 5\% skimmed milk and incubated with anti-p53 antibody (Calbiochem, La Jolla, CA, USA) or monoclonal anti-GADD45 antibody (Santa Cruz Biotechnology, Santa Cruz, CA, USA) in 3\% skimmed milk in PBS for $1 \mathrm{~h}$ followed by horseradish peroxidase-conjugated secondary antibody. The levels of p53 and GADD45 were analyzed for visualization by the ECL system (Amersham Life Science, Amersham, Bucks, U.K.).

\section{Loss of mitochondrial membrane potential and detection of cytochrome $\mathrm{c}$ release}

To check the depolarized population, cells were labeled with mitochondria-specific fluorescent cationic dye MitoTracker RedCMX Ros (Molecular Probes, Eugene, OR, USA). MitoTracker RedCMX Ros was added in culture media at a concentration of $100 \mathrm{nM}$ for $30 \mathrm{~min}$ at $37^{\circ} \mathrm{C}$ and analyzed by FACScan flow cytometer (Becton Dickinson, San Jose, CA, USA). In order to detect the release of the mitochondrial cytochrome $\mathrm{c}$, cells were fractionated as described (Gross et al., 1998) with some modifications as follows. Cells were washed twice with PBS-EDTA (Sigma, St Louis, MO, USA) and lysed in $500 \mu \mathrm{l}$ of isotonic buffer (10 mM HEPES, 250 mM sucrose, 1 mM EDTA, 1 mM EGTA, 1 mM DTT) containing $2 \mathrm{mM}$ of phenyl methyl sulfonyl fluoride
(PMSF) and $100 \mu \mathrm{g}$ of leupeptin per $\mathrm{ml}$. Scrapped cells were lysed by Dounce homogenizer (Pyrex) and centrifuged at $600 \mathrm{~g}$ for $10 \mathrm{~min}$, then supernatant was resuspended with $\mathrm{HM}$ buffer $(10 \mathrm{mM}$ Hepes, $5 \mathrm{mM}$ $\mathrm{MgCl}_{2} 42 \mathrm{mM} \mathrm{KCl}, 1 \%$ Triton X-100), centrifuged at $15,000 \mathrm{~g}$ for $5 \mathrm{~min}$, and pellet (mitochondrial fraction) was dissolved with isotonic buffer. The supernatant fraction was recovered, centrifuged at $300,000 \mathrm{~g}$ for $1 \mathrm{~h}$, and used as cytoplasmic fraction. Protein concentration of each cell fraction was determined by Bio-Rad protein assay (Bio-Rad Laboratories, Hercules, CA, USA) and $50 \mu \mathrm{g}$ of protein was loaded into each lane and separated using $12 \%$ SDS-PAGE and electrophoretically transferred to an Immobilon-P membrane. The membrane was then blocked with PBS containing 3\% skim milk and incubated with rabbit anticytochrome c antibody (1 $\mu \mathrm{g} / \mathrm{ml})$ (Santa Cruz Biotechnology) in 3\% skimmed milk in PBS solution for $1 \mathrm{~h}$ followed by a horseradish peroxidase-conjugated secondary antibody. The level of cytochrome $c$ was analyzed for visualization by the ECL system (Amersham Life Science). All these experiments related with mitochondrial dysfunction were carried out at least in triplicate.

\section{Caspase activity assay using fluorescent substrates}

Cells $\left(1 \times 10^{7}\right.$ cells) were collected at the indicated time points, washed with PBS, frozen in liquid $\mathrm{N}_{2}$, thawed immediately and resuspended in an isolation buffer (20 $\mathrm{mM}$ Hepes, $10 \mathrm{mM} \mathrm{KCl}, 0.5 \mathrm{mM}$ Na-EDTA, $2 \mathrm{mM}$ 2mercaptoethanol, $0.1 \mathrm{mM}$ PMSF, $10 \mu \mathrm{g} / \mathrm{ml}$ leupeptin, 25 $\mu \mathrm{g} / \mathrm{ml}$ ALLN, pH 7.6) as described (Kim et al., 2000). After incubation for $10 \mathrm{~min}$ at $4^{\circ} \mathrm{C}$, the cells were disrupted by 20-30 strokes with a homogenizer and clarified by centrifugation for $1 \mathrm{~h}$ at $100,000 \mathrm{~g}$ as described (Liu et al., 1996). The resulting supernatants were stored at $70^{\circ} \mathrm{C}$. Enzymatic reactions were carried out at $37^{\circ} \mathrm{C}$ for $30 \mathrm{~min}$ in a reaction buffer $(0.1 \mathrm{M}$ HEPES, 2 mM Dithiothreitol, $0.1 \%$ Chaps, $1 \%$ Sucrose) containing $100 \mu \mathrm{g}$ protein and $50 \mu \mathrm{M}$ DEVD-AMC, or 50 $\mu M$ LEHD-AMC. The fluorescent AMC formation was measured emission at $480 \mathrm{~nm}$ with excitation at $360 \mathrm{~nm}$ with a FL600 fluorescence microplate reader (Bio-TEK, Burlington, VT, USA).

\section{Statistical analysis}

ANOVA using Fisher's least significant difference was used. Data were expressed as the mean \pm SEM.

\section{Results}

\section{Effect of HPV 16 E6 antisense nucleic acid on E6 splicing}

To test the effect of HPV 16 E6 antisense nucleic acid 


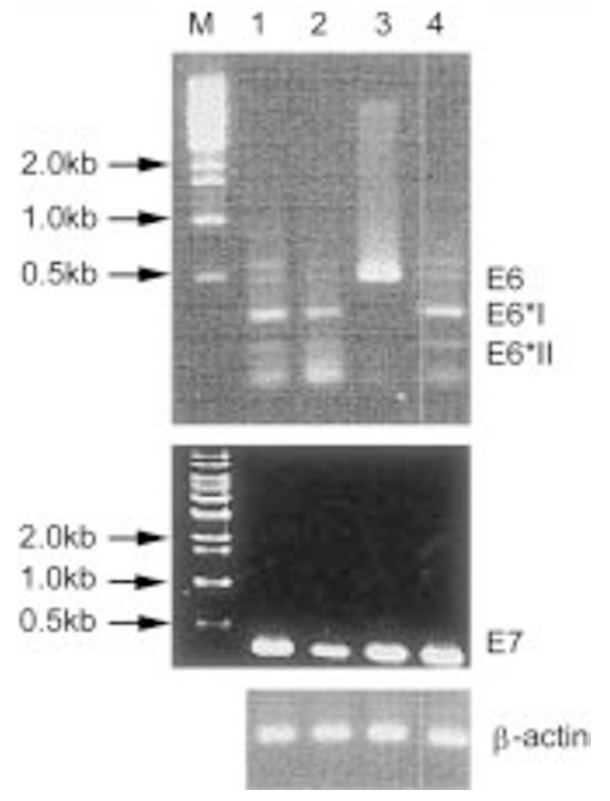

Figure 1. Levels of endogenous HPV 16-specific E6 and E7 transcripts from total RNA extracted from CaSki cell lines transfected with E6(AS) by RT-PCR. The RT-PCR was performed as described in Materials and Methods; M, molecular marker (1kb ladder); lane 1, CaSki cells; lane 2, CaSki cells treated with pTarget vector alone; lane 3, CaSki cells treated with E6(AS); lane 4, CaSki cells treated with E6(S).

on the transcription of E6 and the viability of cervical cancer cells, cervical carcinoma cells were transfected with HPV 16 E6(AS) constructed in pTarget plasmid, respectively. Transcripts of E6 and E7 were detected in CaSki cells after treatment of antisense nucleic acids of HPV 16 by RT-PCR (Figure 1). When CaSki cells were transfected with E6(AS), the spliced forms of E6 transcripts, E6*I and E6*II, were not detected (Figure 1, lane 3), implying that E6 splicing was inhibited by E6(AS) and spliced transcripts E6*I and E6*II might play a critical role in the development as well as in the maintenance of malignant cervical carcinomas. The spliced forms of E6 transcripts, E6 ${ }^{\star} I$ and E6*II, were not inhibited by E6(S) (Figure 1, lane 4). In order to detect E6 expression in E6(AS)-transfected cells, a Dot ELISA was performed by using anti-E6 antibody and nitrocellulose membrane as described (Le Buanec et al., 1999; Lee SJ et al., 2001;). The result of Dot-ELISA showed that there were no significant changes in the expressed level of HPV E6 protein in E6(AS)-transfected CaSki cells (data not shown). However, the viability of cells was suppressed by HPV E6 antisense. The possible explanations of this result are, first, the expressed E6 protein was usually unstable and might hinder the detection of the expressed level of E6 protein altered in E6(AS)-transfected cells, secondly, the transfection of E6 antisense only induced the change of splicing pattern of E6 and the decrease of
A

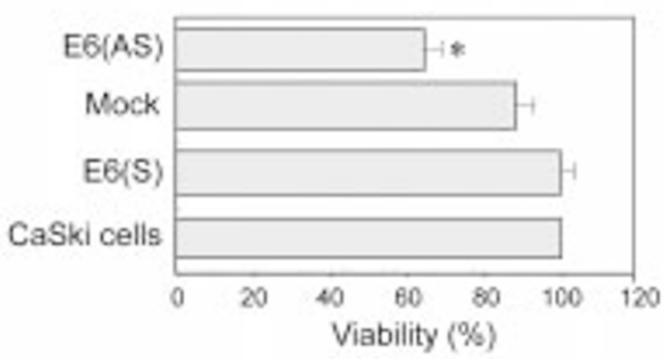

B
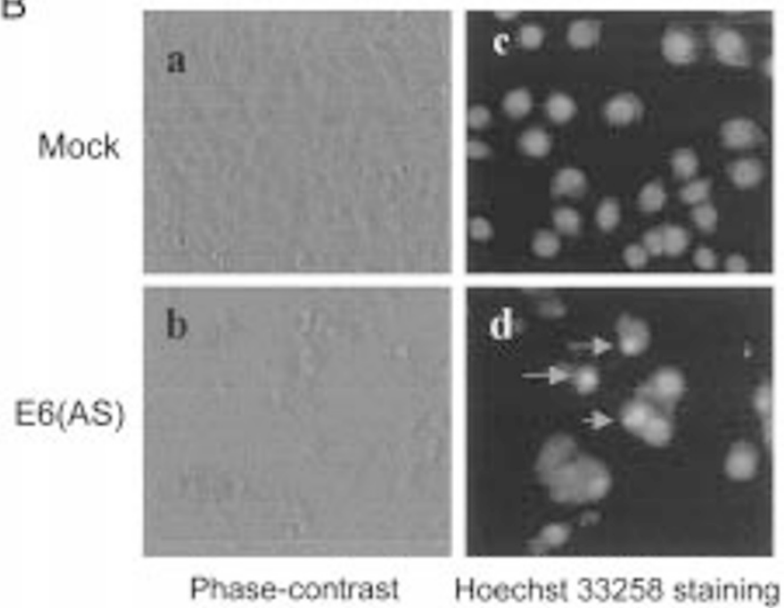

Figure 2. The viability of cervical carcinoma cell lines transfected with E6(AS) (A) and the analysis of apoptosis in CaSki cells after transfection with E6(AS) by Hoechst staining (B). A. Cervical carcinoma CaSki cells were transfected with E6(AS) and were plated on a 96-well plate. E6(S) was used as a sense control vector. Two days after transfection, $10 \mu \mathrm{l}$ of cell proliferation reagent WST-1 was added to the cells and incubated at $37^{\circ} \mathrm{C}$ for $2 \mathrm{~h}$, and then the optical density was measured at $450 \mathrm{~nm}-650 \mathrm{~nm}$. The experiment was done in triplicate and repeated three times. $P^{*}<0.05$ compared to CaSki cells or mock using ANOVA. B. CaSki cells were observed after transfection with E6(AS) (original magnification X400). CaSki cells were seeded in 6 well plates, grown overnight and transfected with pTarget (a) or pTarget/E6(AS) (b) and observed under phase-contrast microscope. Hoechst 33258 staining was performed to identify the chromosome condensation and degradation using an Axiovert fluorescence microscope (Zeiss, Germany) as described in Materials and Methods. CaSki cells were treated with pTarget vector alone (c) and E6(AS) (d).

E6 spliced forms such as E6 $6^{*}$ and E6*Il leads to the increased stability of p53 and apoptosis in E6(AS)transfected CaSki cells.

\section{Effect of HPV 16 E6 antisense nucleic acid on cell viability and apoptosis}

The cell viability of CaSki cells was inhibited by $30 \pm 5 \%$ compared to mock control within 2 days after transfection of E6(AS) (Figure 2A). However, there was little effect of E6(AS) on the viability of C-33A cells (data not shown), which do not harbor HPV DNA. The viability of HeLa cells which contain HPV 18 genome was not significantly changed after transfection with E6(AS) 
A
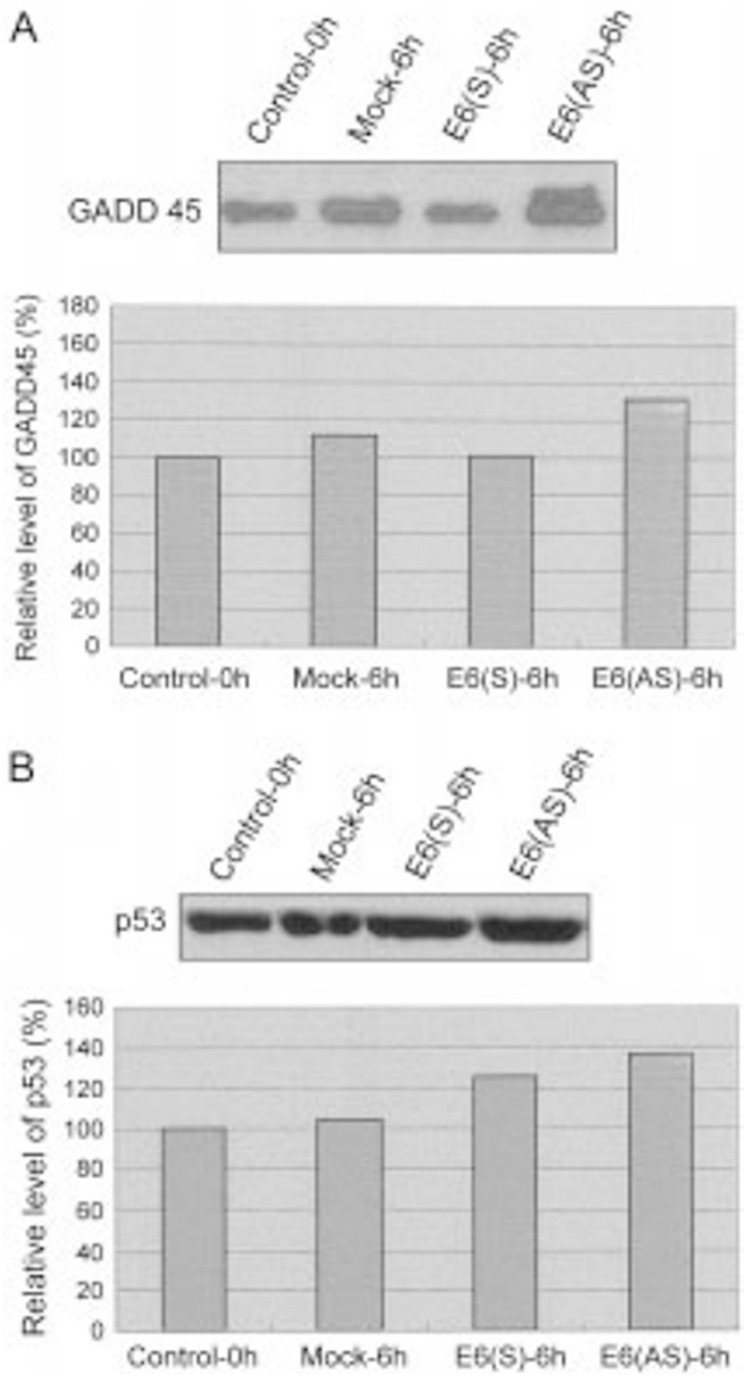

Figure 3. Detection of expression levels of p53 and GADD45 in CaSki cells after treatment with E6(AS). Equal amounts of cell lysates were loaded into each lane and separated using SDS-PAGE and electrophoretically transferred to an Immobilon-P membrane. The membrane was then blocked with 5\% skimmed milk in PBS and incubated with anti-p53 antibody (A) or anti-GADD45 antibody (B) for $1 \mathrm{~h}$ followed by horseradish peroxidaseconjugated secondary antibody. The levels of p53 and GADD45 were analyzed for visualization by the ECL system (Amersham Life Science, Amersham, Bucks, UK).

(data not shown), suggesting that the effect of E6(AS) of HPV 16 was specific to the cells containing E6 sequences of HPV type 16. The mock (pTarget vector) or E6 sense control [E6 sense nucleic acid ; E6(S)] did not affect on the viability of any cells we tested. The morphology of CaSki cells was changed to be round up and detached from the surface of the culture plate within 2 days after the transfection with pTarget/E6(AS) (Figure 1B-b) compared with that of CaSki cells transfected with pTarget (Figure 1B-a). The similar results were obtained in the E6(AS) transfected SiHa cells (data not shown). However, neither C-33A nor HeLa cells were morpho- logically altered in the presence of E6(AS) (data not shown). The results demonstrate that E6(AS) affected the viability and morphology of only the certain cervical carcinoma cell lines, CaSki and SiHa cells which harbor HPV 16 genome. To analyze whether the inhibition of cell proliferation induced by E6 (AS) is caused by apoptosis, E6 (AS) transfected cells were stained with Hoechst 33258. As shown in Figure 1B-c and d, chromosome condensation and degradation were observed in only the CaSki cells transfected with E6 (AS) but not in pTarget transfected control cells. These results clearly demonstrated that transfection of E6 (AS) induced apoptosis in CaSki cells.

\section{Upregulation of p53 by E6(AS)}

In order to detect the effect of E6 (AS) on the level of p53, Western blotting experiment was performed and the result showed that the protein level of p53 was increased upto about $35 \%$ in E6 (AS)-transfected cells compared to mock-control cells (Figure $3 A$ ). To elucidate whether p53 protein upregulated by E6 (AS) transfection was active enough to induce, a p53-regulated target gene GADD45, the protein level of GADD45 was detected. The result of Western blotting shows that the level of GADD45 protein was also upregulated upto about $20 \%$ in E6(AS) transfected CaSki cells compared to mock-control cells (Figure 3B). These results showed that E6(AS) plays a role in inhibition of E6 splicing and upregulation of p53 protein; It has been well known that p53 is degradaded by E6 oncogene via ubiquitination pathway (Lechner et al., 1994; Beer-Romero et al., 1997). E6(AS) also inhibited the spliced forms of E6 transcripts, E6*I and E6*II (Figure 1, lane 3) which might play a critical role in the development of malignant cervical carcinomas. Therefore, it is possible to speculate that down regulation of E6 expression by antisense delivery is able to enhance the stabilization of $p 53$.

\section{Mitochondrial depolarization, cytochrome c release, and caspase activation}

Then we investigated whether the upregulated p53 could induce the apoptosis specifically related with mitochondrial dysfunction. The depolarized population of mitochondria was measured by staining CaSki cells with the mitochondria-specific fluorescent cationic dye and analyzed by FACScan flowcytometer. To detect the mitochondrial membrane potential, cells were labeled with MitoTracker RedCMX Ros (100 nM). The population of decreased MitoTracker RedCMX Ros fluorescence (18.5\%) was observed in E6(AS)-transfected cell lines within $6 \mathrm{~h}$ after transfection compared with control E6

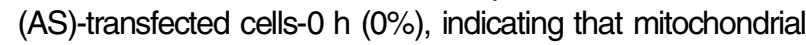
membrane potential was decreased in these E6(AS)treated CaSki cervical cancer cells (Figure 4A). Cytoplasmic and mitochondrial fractions were collected 
A
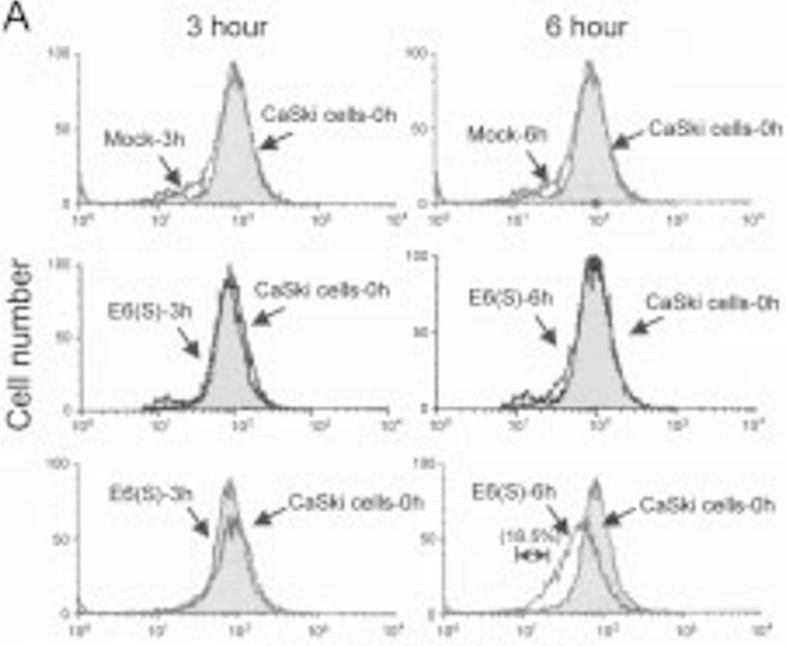

Fluorescence Intensity

B

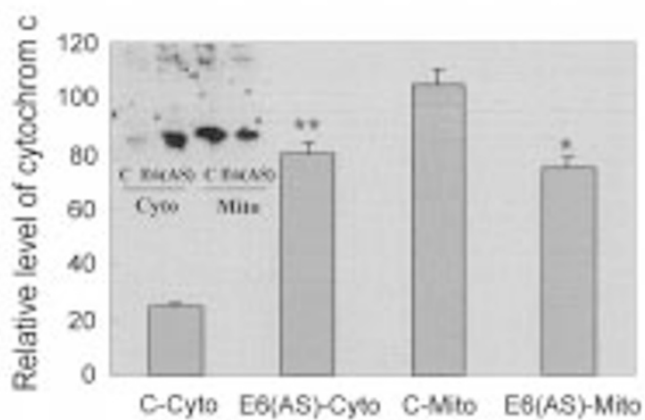

Figure 4. Detection of loss of mitochondrial membrane potential and cytochrome $c$ release from membrane in E6(AS)-transfected CaSki cells. Cells were labeled with mitochondria-specific fluorescent cationic dye MitoTracker RedCMX Ros. The dye was added in culture for $30 \mathrm{~min}$ at $37^{\circ} \mathrm{C}$ and analyzed by FACScan flow cytometer (A). Fifty $\mu \mathrm{g}$ of protein was loaded into each lane and analyzed on SDS-PAGE and electrophoretically transferred to an Immobilon-P membrane. The membrane was then blocked and incubated with rabbit anti-cytochrome $c$ antibody followed by a horseradish peroxidase-conjugated secondary antibody. The level of cytochrome $c$ was visualized by the ECL system (Amersham Life Science, Inc.) and analyzed by densitometer. Cyto stands for cytoplasmic fraction and Mito stands for mitochondrial fraction. $P^{* *}<0.01$ campared to E6(AS) transfectant with mock in cytoplasmic fraction and $P^{*}<0.01$ compared to E6(AS) transfectant with mock in mitochondrial fraction using ANOVA(B).

using CaSki cells at $6 \mathrm{~h}$ after transfection. Then equal amounts of proteins were fractionated in SDS-PAGE and Western blot analysis of cytochrome $\mathrm{C}$ was performed. These results showed that the amount of cytochrome $\mathrm{c}$ was increased in cytoplasmic extracts whereas decreased in mitochodrial fraction in E6(AS)transfected cell lines compared to mock-transfected cells (Figure 4B), showing that cytochrome c was released from mitochodrial membrane into cytosol. Caspases play a central role in mediating various apoptotic responses. Hence E6(AS) induced cytochrome $c$ release, the enzymatic activities of
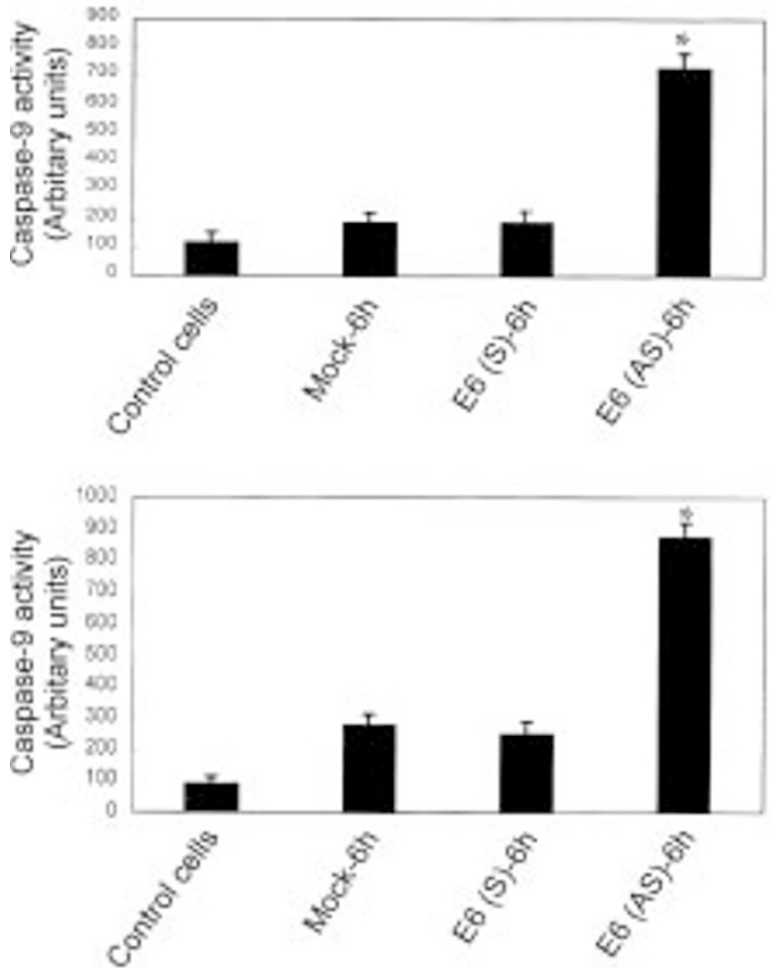

Figure 5. Detection of activities of caspase-3 and caspase-9 in CaSki cells after treatment with E6(AS). Caspase activation by E6(AS) treatment was examined by assaying enzyme activities using fluorogenic substrates. Enzymatic reactions were carried out at $37^{\circ} \mathrm{C}$ for $30 \mathrm{~min}$ in a reaction buffer containing $100 \mu \mathrm{g}$ of protein and $50 \mu \mathrm{M}$ DEVD-AMC, or $50 \mu \mathrm{M}$ LEHD-AMC. The fluorescent AMC formation was measured emission at $480 \mathrm{~nm}$ with excitation at $360 \mathrm{~nm}$ using a FL600 fluorescence microplate reader. $P^{*}<0.05$ compared to CaSki cells or mock using ANOVA.

caspases which are the downstream effectors of cytochrome $\mathrm{c}$, were assessed by using two fluorogenic peptide substrates (Ac-DEVD-AMC for caspase-3 and Ac-LEHD-AMC for caspase-9). Caspase activities were measured $6 \mathrm{~h}$ after E6(AS) transfection. It was shown that the activities of caspase- 3 and -9 were enhanced by about 3-folds in E6(AS)-transfected CaSki cells compared to mock-transfected or sense control cells (Figure 5). These results clearly demonstrated that the upregulated p53 protein which may be caused by inhibition of splicing of E6 and stabilization of p53 in E6(AS)-transfected CaSki cells are functional and mitochondrial dysfunction and caspase cascades may be sequentially activated to induce apoptosis.

\section{Discussion}

To maximize the specificity and efficiency of the antisense nucleic acid effect, we chose the CaSki cells which contain a 500 600 copies of integrated HPV 16 genome compared with $\mathrm{SiHa}$ cells containing only 1-2 
copies of HPV 16 (Baker et al., 1987; Meissner, 1999). It was reported that CaSki cells contain $60-600$ copies of HPV 16 while HeLa cells harbor only $10-50$ copies of HPV 18 by which carcinoma is induced (Schwarz et al., 1985). Even though mRNAs encoding E6 and E7 of HPVs are monocistronic (Chow et al., 1987; Smotkin et al., 1989), the splicing pattern for the early viral transcripts of the oncogenic types, such as HPV 16 and HPV 18, is complex. In most of cervical tumor samples, full-length E6 exists in extremely low abundance and most of transcripts of E6 was found as E6*I and the other spliced form E6*II was rarely detected (Bohm et al., 1993; Grassmann et al., 1996). In E6(AS) transfected Caski cells, the E6 splicing pattern was checked. E6 $6^{\star}$ and E6*II were hardly detected and only full-length E6 was detected. Our results is consistent with previous reports (Bohm et al., 1993; Grassmann et al., 1996), suggesting that E6*I may have a critical role in the development and maintenance of cervical carcinomas.

E6 and E7 were reported to regulate cell proliferation by reducing the activity of tumor suppressor proteins, p53 and pRb (Cho et al., 2001), respectively. Thus, antisense of E6 or E7 might be expected to affect the cell cycle or cell death. In the present study, E6(AS) specifically affected the viability and morphology of the cervical carcinoma cell lines, CaSki and SiHa cells that harbor HPV 16 genome. Also, E6(AS) induced apoptosis in CaSki cells. It has been demonstrated that E6 protein forms complexes with p53 (Storey et al., 1998) and can target p53 for degradation through the ubiquitin pathway (Lechner et al., 1994; Beer-Romero et al., 1997). The reduced level of the spliced E6, E6*I and E6*II may cause the enhanced stability of p53. Thus, E6(AS) had a role in suppressing the transcription of the spliced forms of E6 and the growth of cervical epithelial carcinoma cells. Slight increase of p53 in E6(S)transfected cells may be due to the nonspecific effect of E6 nucleic acid expression. It was reported that stranded DNA oligonucleotides activate p53 by the interaction with a nonspecific DNA binding domain of p53 (Hadshiew et al., 2001). The MPT is an event reported to be important in activation of apoptotic cell death and a loss of the mitochondrial potential $(\triangle \varphi m)$ is induced by the change of permeability associated with MPT (Heiskanen et al., 1999). There have been no reports to show the mitochondrial dysfunction during the apoptotic process induced by E6 antisense transfection. In current study, we observed decrease in mitochondrial membrane potential in E6(AS)-transfected cell lines within $6 \mathrm{~h}$ after transfection relatively early stage of apoptosis, showing that cytochrome $\mathrm{c}$ was released from mitochondrial membrane into cytosol. In addition, the activities of caspase-3 and 9 which are the downstream effectors of cytochrome c, were also enhanced by about 3-folds in E6(AS)-transfected CaSki cells compared to mock-transfected cells. From these results, we suggest that antisense E6 nucleic acid may induce upregulation of active p53 which leads to the enhancement of p53-regulated target gene Gadd45, and cytochrome $c$ release from mitochondrial membrane. This study also could provide a novel in vitro evidence that caspase- 9 and caspase- 3 , which are conventionally known for the downstream effectors in apoptosis, are also activated by the introduction of E6(AS) into CaSki cells, a cervical carcinoma cell line. Deregulation of cell death pathways is an important feature of tumorigenesis. Therefore, our study support one of in vitro evidences that antisense gene therapy applies the techniques of rational drug design to develope a range of drugs that targets a well-characterized gene product. because an antisense oligonucleotides are relatively easy to design, synthesize and have a predictable high affinity for the disease-targeted gene, leading to the minimization of side effects.

\section{Acknowledgments}

This work was supported by grant Molecular Medicine Program (M1-0106-00-0078) from the Ministry of Science and Technology through the Biomedical Research Center at KAIST and in part from Ministry of Education.

\section{References}

Baker CC, Phelps WC, Lindgren V, Braun MJ, Gonda MA, Howley PM. Structural and transcriptional analysis of human papillomavirus type 16 sequences in cervical carcinoma cell lines. J. Virol. 1987;61:962-71.

Beer-Romero P, Glass S, Rolfe M. Antisense targeting of E6AP elevates p53 in HPV-infected cells but not in normal cells. Oncogene 1997; 14:595-602.

Bohm S, Wilczynski SP, Pfister H, Iftner T. The predominant mRNA class in HPV16-infected genital neoplasias does not encode the E6 or the E7 protein. Int. J. Cancer 1993;55:79198.

Cho YS, Cho CW, Joung O, Lee KA, Park SN, Yoon DY. Development of screening systems for drugs against human papillomavirus-associated cervical cancer: based on E6-E6AP binding. Antiviral Res. 2000;47:199-206.

Cho YS, Cho CW, Kang JW, Cho MC, Lee KA, Shim JH, Kwon DH, Choe YK, Yoon DY. Development of a screening system for drugs against human papillomavirus-associated cervical cancer: Based on E7-Rb binding. J. Biochem. Mol. Biol. 2001;34:80-84.

Chow LT, Nasseri M, Wolinsky SM, Broker TR Human papillomavirus types 6 and 11 mRNAs from genital condylomata acuminata. J. Virol. 1987;61:2581-88.

Grassmann K, Rapp B, Maschek H, Petry KU, Iftner T. 
Identification of a differentiation-inducible promoter in the E7 open reading frame of human papillomavirus type 16 in raft cultures of a new cell line containing high copy numbers of episomal HPV-16 DNA. J. Virol. 1996;70:2339-49.

Gross A, Jockel J, Wei MC, Korsmeyer SJ. Enforced dimerization of BAX results in its tanslocation, mitochondrial dysfunction and apoptosis. EMBO. J. 1998;17:3878-85.

Hadshiew IM, Eller MS, Gasparro FP, Gilchrest BA. Stimulation of melanogenesis by DNA oligonucleotides: effect of size, sequence and 5' phosphorylation. J. Dermatol. Sci. $2001 ; 25(2): 127-38$

Hamada K, Sakaue M, Alemany R, Zhang W, Horio Y, Roth JA, Mitchell MF. Adenovirus-mediated transfer of HPV 16 E6/ E7 antisense RNA to human cervical cancer cells. Gynecologic Oncology 1996;63:219-27.

He Y, Huang L. Growth inhibition of human papillomavirus 16 DNA-positive mouse tumor by antisense RNA transcribed from U6 promoter. Cancer Research 1997;57:3993-99.

Heiskanen KM, Bhat MB, Wang HW, Ma J. Nieminen AL. Mitochondrial depolarization accompanies cytochrome c release during apoptosis in PC6 cells. J. Biol. Chem. 1999;274:5654-58.

Kastan MB, Zhan Q, El-Deiry WS, Carrier F, Jacks T, Walsh, WV, Plunkett BS, Vogelstein B, Fornace AJ, Jr. A mammalian cell cycle checkpoint pathway utilizing p53 and GADD45 in defeective in ataxia-telangiectasia. Cell 1992;71:587-97.

Kim IK, Chung CW, Woo HN, Hong GS, Nagata S, Jung YK. Regulation of caspase-8 sensitizes JB6 cells to TRAIL. Biochem. Biophys. Res. Comm. 2000;277:311-16.

Le Buanec H, Lachgar A, D'Anna R, Zagury JF, Bizzini B, Bernard J, Ittele D, Hallez S, Giannouli C, Burny A, Zagury D. Induction of cellular immuno-suppression by the human papillomavirus type 16 E7 oncogenic protein. Biomed Pharmacother 1999;53:323-28.

Lechner MS, Laimins LA Inhibition of p53 DNA binding by human papillomavirus E6 proteins. J. Virol. 1994;68:4262-73.

Lee SJ, Cho YS, Cho MC, Cho CW, Shim JH, Lee KA, Ko KK, Choe YK, Park SN, Kim SH, Dinarello S, Yoon DY. Both E6 and E7 oncoproteins of HPV 16 inhibited the IL-18 induced
IFN-g production in immune cells via competitive binding to IL18 receptor. J Immunol. 2001;167: 497-504.

Liu X, Kim CN, Yang J, Jemmerson R. Wang XInduction of apoptotic program in cell-free extracts requirements for dATP and cytochrome c. Cell 1996;86:147-57.

Madrigal M, Janicek MF, Sevin B, Perras J, Estape, R, Penalver $\mathrm{M}$, Averette HE. In vitro antigene therapy targeting HPV-16 E6 and E7 in cervical carcinoma. Gynecologic Oncology 1997;61:18-25.

Meissner JD. Nucleotide sequences and further characterization of human papillomavirus DNA present in the CaSki, SiHa and HeLa cervical carcinoma cell lines. Gen. Virol. 1999;80:1725-33.

Paoletti C. Anti-sense oligonucleotides as potential antitumour agents: prospective views and preliminary results. Anticancer Drug Des. 1988;2:325-31.

Schwarz E, Freese UK, Gissmann L, Mayer W, Roggenbuck, $B$, Stremlau A, zur Hausen $H$. Structure and transcription of human papillomavirus sequences in cervical carcinoma cells. Nature 1985;314:111-14.

Sheffner M, Munger K, Byrne JC, Howley PM. The state of the p53 and retinoblastoma genes in human cervical carcinoma cell lines. Proc. Natl. Acad. Sci. USA. 1991;88: 5523-27.

Smotkin D, Prokoph H, Wettstein FO. Oncogenic and nononcogenic human genital papillomaviruses generate the E7 mRNA by different mechanisms. J. Virol. 1989;63:1441-47.

Storey A, Thomas M, Kalita A, Harwood C, Gardiol D, Mantovani F, Breuer J, Leigh IM, Matlashewski G, Banks L. Role of a p53 polymorphism in the development of human papillomavirus- associated cancer. Nature 1998;393: 229-34.

Sun XM, MacFarlane M, Zhuang J, Wolf BB, Green DR, Cohen GM. Distinct caspase cascades are initiated in receptor-mediated and chemical-induced apoptosis. J. Biol. Chem. 1999;274:5053-60.

Tan TM, Ting RC. In vitro and in vivo inhibition of human papillomavirus type 16 E6 and E7 genes. Cancer Research 1995;55:4599-605. 